\title{
Euclidean Methods and the entropy function
}

\author{
Pedro J. Silva, \\ Institut de Ciències de l'Espai (IEEC-CSIC) and \\ Institut de Física d'Altes Energies (IFAE), \\ E-08193 Bellaterra (Barcelona), Spain \\ psilva@ifae.es \\ Received 15 April 2008, Published in Fortschr. Phys. 56, No 7 - 9, 856 - 861 (2008)
}

\begin{abstract}
We review results of articles hep-th/0607056, hep-th/0610163 and 0704.1405 [hep-th]. Here we focus on establish the connection between the entropy functional formalism of Sen and the standard Euclidean formalism taken at zero temperature. We find that Sen's entropy function $f$ (on-shell) matches the zero temperature limit of the Euclidean action. Moreover, Sen's near horizon angular and electric fields agree with the chemical potentials that are defined from the zero-temperature limit of the Euclidean formalism. Connection with the Dual CFT thermodynamics is briefly discussed.
\end{abstract}

\section{Introduction}

This article contains the talk based on the articles [1, 2, 3]. Here we only show the main results and general lessons that steam from our work. More References and more details should be found on our original papers.

\section{On Sen's entropy functional formalism}

Black holes $(\mathrm{BH})$ are one of most interesting laboratories we have to investigate quantum gravity effects. Due to their thermodynamic behavior these objects have been associated to ensembles of microstates in the fundamental quantum gravity theory where ideally, quantum statistical analysis should account for all the BH coarse-grained thermodynamical behavior. In particular, many important insights in the classical and quantum structure of $\mathrm{BH}$ have been obtained studying supersymmetric configurations in string theory. In this context we have the so called attractor mechanism. It was originally thought in the context of four dimensional $N=2$ supergravity, where we have that the values of the scalar fields at the horizon are given by the values of the $\mathrm{BH}$ conserved charges and are independent of the asymptotic values of the scalars at infinity.

Importantly, the attractor mechanism has provided a new way to calculate the BH entropy. In a series of articles [4, 5, 6], Sen recovered the entropy of $D$-dimensional BPS BH using only the near horizon part of the geometry. Basically, in this regime the solution adopts the form $A d S_{2} \otimes S^{D-2}$ 
1 plus some electric and magnetics fields. The entropy $S$ is obtained by introducing a function $f$ as the integral of the corresponding supergravity Lagrangian over the $S^{D-2}$. More concretely, an entropy function is defined as $2 \pi$ times the Legendre transform of $f$ with respect to the electric fields $e_{i}$. Then, an extremization procedure fixes the on-shell BPS values of the different fields of the solution and in particular determines the BPS value of the entropy $S$,

Sen's entropy functional formalism assumes that: (i) we start with a Lagrangian $\mathcal{L}$ with gravity plus some field strengths and uncharge massless scalar fields; and (ii) due to the attractor mechanism the near horizon geometry of a $D$-dimensional $\mathrm{BH}$ is set to be of the form $A d S_{2} \otimes S^{D-2}$. From the above input data, the general form of the near horizon $\mathrm{BH}$ solution is

$$
\begin{aligned}
& d s^{2}=v_{1}\left(-\rho^{2} d \tau+\frac{d \rho^{2}}{\rho^{2}}\right)+v_{2} d \Omega_{D-2}^{2}, \\
& F_{\rho \tau}^{(i)}=e_{i}, \quad H^{(a)}=p_{a} \epsilon_{D-2}, \\
& \phi_{s}=u_{s},
\end{aligned}
$$

where $\epsilon_{D-2}$ is the unit-volume form of $S^{D-2}$, and $\left(e_{i}, p_{a}\right)$ are respectively the electric fields and the magnetic charges of the $\mathrm{BH}$. Note that $(\vec{u}, \vec{v}, \vec{e}, \vec{p})$ are arbitrary constants up to now and therefore the solution is off-shell. Next, it is defined the following function

$$
f(\vec{u}, \vec{v}, \vec{e}, \vec{p})=\int_{S^{D-2}} \sqrt{-g} \mathcal{L},
$$

where $\mathcal{L}$ is the string frame Lagrangian of the theory. After minimizing $f(\vec{u}, \vec{v}, \vec{e}, \vec{p})$ with respect to $(\vec{u}, \vec{v})$ we obtain the exact supersymmetric near horizon $\mathrm{BH}$ solution in terms of $(\vec{e}, \vec{p})$. In fact, the field equations are reproduced by this minimization procedure. Furhermore, minimization with respect to $\vec{e}$ gives the electric charges $\vec{q}$. Explicitly, the on-shell values of $\vec{u}, \vec{v}, \vec{e}$ that specify (11) for a given theory described by (2) are found through the relations,

$$
\frac{\partial f}{\partial u_{s}}=0, \quad \frac{\partial f}{\partial v_{j}}=0, \quad \frac{\partial f}{\partial e_{i}}=q_{i} .
$$

Then, using Wald formalism [7], Sen derived that the entropy $S$ of the corresponding BH is given by $2 \pi$ times the Legendre transform of $f$,

$$
S=2 \pi\left(e_{i} \frac{\partial f}{\partial e_{i}}-f\right)
$$

Finally notice that the minimization procedure, can be taken only after $S$ is defined. In this form $S$ is really an entropy function of $(\vec{u}, \vec{v}, \vec{q}, \vec{p})$, that after minimization equals the BH entropy as a function of $(\vec{q}, \vec{p})$ only.

The above formalism fixes the form of the NH geometry and the entropy $S$ in terms of the conserved charges but what is the geometric origin or motivation for the above definitions? and how is connected to the usual Bh thermodynamics? To answer these questions we revisit the Bh thermodynamics and the limit of zero temperature in next section.

\footnotetext{
${ }^{1}$ The analysis of the near horizon geometry has been applied to more general BH that define squashed $A d S_{2} \otimes S^{D-2}$ geometries.
} 


\section{On GR thermodynamics and zero temperature limit}

In 1, 2] the "thermodynamics" or better "the statistical mechanics" of supersymmetric solitons in gauged supergravity was studied in detail using an extension of standard Euclidean thermodynamical methods to zero temperature systems. We call this approach the Euclidean zero-temperature formalism. BPS BH can be studied as dual configurations of supersymmetric ensembles at zero temperature but non-zero chemical potentials in the dual CFT. These potentials control the expectation value of the conjugated conserved charges carried by the $\mathrm{BH}$, like e.g., angular momenta and electric charge.

In these articles, the two main ideas are: First, there is a supersymmetric field theory dual to the supergravity theory. Second, in this dual field theory the grand canonical partition function over a given supersymmetric sector can be obtained as the zero temperature limit of the general grand canonical partition function at finite temperature. This limit also fixes the values of several chemical potentials of the system.

To make things more clear, recall that all supersymmetric states in a field theory saturate a BPS inequality that translates into a series of constraints between the different physical charges. For definiteness, let us consider a simple case where the BPS bound corresponds to the constraint: $E=J$. Then, defining the left and right variables $E^{ \pm}=\frac{1}{2}\left(E_{\nu} \pm J_{\nu}\right), \beta_{ \pm}=\beta(1 \pm \Omega)$ the grand canonical partition function is given by

$$
Z_{(\beta, \Omega)}=\sum_{\nu} e^{-\left(\beta_{+} E_{+}+\beta_{-} E_{-}\right)} .
$$

At this point, it is clear that taking the limit $\beta_{-} \rightarrow \infty$ while $\beta_{+} \rightarrow \omega$ (constant), gives the correct supersymmetric partition function. The above limiting procedure takes $T$ to zero, but also scales $\Omega$ in such a way that the new supersymmetric conjugated variable $\omega$ is finite and arbitrary. Note that among all available states, only those that satisfy the BPS bound are not suppress in the sum, resulting in the supersymmetric partition function

$$
Z(\omega)=\sum_{b p s} e^{-\omega J}
$$

where the sum is over all supersymmetric states (bps) with $E=J$. The above manipulations are easy to implement in more complicated supersymmetric field theories like, e.g., $N=4$ SYM theory in four dimensions. What is less trivial is that amazingly it could also be implemented in the dual supersymmetric configurations of gauged supergravity, since it means that these extreme BPS solutions are somehow protected from higher string theory corrections.

To apply the Euclidean zero-temperature formalism to concrete black hole systems, it is profitable to highlight its key steps. To study the statistical mechanics of supersymmetric black holes we take the off-BPS BH solution and we send $T \rightarrow 0$. In this limiting procedure, the angular velocities and electric potentials at the horizon can be written as an expansion in powers of the temperature. More concretely one has when $T \rightarrow 0$,

$$
\beta \rightarrow \infty, \quad \Omega \rightarrow \Omega_{b p s}-\frac{\omega}{\beta}+O\left(\beta^{-2}\right), \quad \Phi \rightarrow \Phi_{b p s}-\frac{\phi}{\beta}+O\left(\beta^{-2}\right),
$$

where $\beta$ is the inverse temperature; $(\Omega, \Phi)$ are the angular velocities and electric potentials at the horizon; the subscript bps stands for the values of these quantities in the on-shell BPS solution; and 
$(\omega, \phi)$ are what we call the supersymmetric conjugated potentials, i.e., the next to leading order terms in the expansion. For all the systems studied, we find that the charges have the off-BPS expansion,

$$
E=E^{b p s}+\mathcal{O}\left(\beta^{-2}\right), \quad Q=Q^{b p s}+\mathcal{O}\left(\beta^{-2}\right), \quad J=J_{\phi}^{b p s}+\mathcal{O}\left(\beta^{-2}\right),
$$

where $(E, Q, J)$ are the energy, charges and angular momenta of the $\mathrm{BH}$. In supergravity, the grand canonical partition function in the saddle point approximation is related to so called quantum statistical relation (QSR)

$$
I_{(\beta, \Phi, \Omega)}=\beta E-\Phi Q-\Omega J-S,
$$

where $S$ is the entropy, and $(\beta, \Phi, \Omega)$ are interpreted as conjugated potentials to $E, Q, J$, respectively. $I$ is the Euclidean action (evaluated on the off-BPS BH solution) that, in this ensemble, depends only on $(\beta, \Phi, \Omega)$. It plays the role of free energy divided by the temperature. Inserting (7) and (8) into (9) yields

$$
I_{(\beta, \Phi, \Omega)}=\beta\left(E^{b p s}-\Phi_{b p s} Q^{b p s}-\Omega_{b p s} J^{b p s}\right)+\phi Q^{b p s}+\omega J^{b p s}-S_{b p s}+\mathcal{O}\left(\beta^{-1}\right) .
$$

Here, we observe that this action is still being evaluated off-BPS. Moreover, the term multiplying $\beta$ boils down to the BPS relation between the charges of the system and thus vanishes (this will become explicitly clear in the several examples we will consider). This is an important feature, since now we can finally take the $\beta \rightarrow \infty$ limit yielding relation

$$
I_{b p s}=\phi Q^{b p s}+\omega J^{b p s}-S_{b p s} .
$$

It is important to stress that this zero temperature limiting procedure yields a finite, not diverging, supersymmetric version of QSR, or shortly SQSR. Note that if we had evaluated the Euclidean action (9) directly on-shell it would not be well defined, as is well-known. As a concrete realization, we picked (and will do so along the paper) the SQSR to exemplify that the $T \rightarrow 0$ limit yields well-behaved supersymmetric relations. The reason being that this SQSR relation is the one that will provide direct contact with Sen's entropy functional formalism, which is the main aim of our study. However, it also provides a suitable framework that extends to the study of the full statistical mechanics of supersymmetric black holes.

\section{On entropy functional and zero temperature thermodynamics}

In previous sections we have described two apparently unrelated procedures to obtain the entropy of supersymmetric BH that naturally contain the definitions of pairs of conjugated variables, related to the $\mathrm{BH}$ charges. In this section we show that both procedures produce basically the same body of final definitions, even though conceptually both approaches are rather different.

That both approaches produce the same final chemical potentials and definitions can be seen in any of the examples at hand. As usual, the best way to illustrate our point is to pick a system that captures the fundamental ingredients. Comparing the zero temperature thermodynamic relations with the corresponding Sen's definitions, in any BH like e.g., the D1-D5-P system, we can indeed confirm that all the key quantities agree in the two formalisms. Explicitly we have that

$$
\phi_{i}=2 \pi e_{i}, \quad Q_{i}=q_{i}, \quad I_{b p s}=2 \pi f .
$$


Nevertheless, that both frameworks are equivalent is a priori not at all obvious since they have important differences. Sen's approach relies completely on the structure of the near horizon geometry. In particular, the entropy is constructed analyzing Wald's prescription and Einstein equations in these spacetimes and all the analysis is carried on at the BPS bound i.e., when the solution is extremal. In contrast, the zero temperature limit approach relies on the thermodynamical properties of $\mathrm{BH}$ and, in principle, uses the whole spacetime, not only the near horizon region. The resulting thermodynamic definitions come as a limiting behavior of non-extremal $\mathrm{BH}$ and have a nice straightforward interpretation in terms of the dual CFT thermodynamics.

\subsection{Near-horizon and asymptotic contributions to the Euclidean action}

To understand why the above close relations between the two formalisms hold, let us go back to the calculation of the Euclidean action for general $\mathrm{BH}$ in the off-BPS regime. Inspired in ten dimensional type II supergravity, we start with the general action 2

$$
I=\frac{1}{16 \pi G} \int_{\Sigma} \sqrt{-g}\left(R-\frac{1}{2}(\partial \Psi)^{2}-\frac{1}{2 n !} e^{\alpha \Psi} F_{(n)}^{2}\right)+\frac{1}{8 \pi G} \int_{\partial \Sigma} K
$$

where $\Sigma$ is the spacetime manifold, $\partial \Sigma$ the boundary of that manifold and $K$ is the extrinsic curvature. In the BH case, once we have switched to Euclidean regime, it is necessary to compactify the time direction to avoid a conical singularity. This compactification defines the Hawking temperature as the inverse of the corresponding compactification radius.

To evaluate the Euclidean action on the $\mathrm{BH}$ solution, one of the methods to obtain a finite result, i.e., to regularize and renormalize the action, consists of putting the $\mathrm{BH}$ in a box and subtract the action of a background vacuum solution $\left(g^{0}, \Psi^{0}, F^{0}\right)$. This procedures also defines the "zero" of all the conserved charges. For asymptotic flat solutions we use Minkowski, while for asymptotic AdS solutions we use AdS. Once in the box, the radial coordinate is restricted to the interval $\left(r_{+}, r_{b}\right)$, where $r_{+}$is the position of the horizon and $r_{b}$ corresponds to an arbitrary point which limits the box and that at the end is sent to infinity. Another important ingredient is the boundary conditions on the box. Basically, depending on which conditions we impose on the different fields, we will have fixed charges or fixed potentials. If we do not add any boundary term to the above action, we will be working with fixed potentials, i.e., we will work in the grand canonical ensemble.

At this point we are ready to rewrite the Euclidean action in two pieces, one evaluated in the first boundary at $r=r_{+}$, and the other in the second boundary at $r=\infty$,

$$
I=\int_{r=r_{+}}\left\{\frac{c}{8 \pi G} e^{a \Psi} F_{(n)} C_{(n-1)}+\frac{1}{8 \pi G} K\right\}+\int_{r=\infty}\left\{\frac{c}{8 \pi G} e^{a \Psi} F_{(n)} C_{(n-1)}+\frac{1}{8 \pi G}\left(K-K^{0}\right)\right\} .
$$

where the field equations have been used and $c$ is a proportionality constant. Then we can rewrite the above expression as,

$$
I=\underbrace{\beta\left(\Phi_{b p s}-\Phi\right) Q-S}_{r=r_{+}}+\underbrace{\beta\left(E-\Phi_{b p s} Q\right)}_{r=\infty} .
$$

\footnotetext{
${ }^{2}$ For simplicity, the reasoning is done at the level of two derivative Lagrangian. Nevertheless, following Wald's approach for higher derivative actions, we notice that the $\mathrm{BH}$ action can always be recast as surface integrals. Moreover, for definiteness, we anchor our discussion to type II action, but whenever needed we make comments to extend our arguments to more general theories.
} 
Therefore we can always find a gauge in which the Euclidean action splits in two contributions, one at the horizon and the other in the asymptotic region. In fact, from our discussion it is easy to see that the first term exactly reproduces the SQSR, i.e.,

$$
\lim _{B P S \text { limit }} \beta\left(\Phi_{b p s}-\Phi\right) Q-S=\phi Q_{b p s}-S_{b p s} .
$$

while the asymptotic term vanishes due to fact that $\Phi_{b p s}=1$, and thus the leading term in the expansion is nothing else than the BPS relation $E_{b p s}=Q_{b p s}$ characteristic of supersymmetric regimes, i.e.,

$$
\lim _{B P S \text { limit }} \beta\left(E-\Phi_{\text {bps }} Q\right)=\lim _{B P S \text { limit }} \beta(E-Q)=0 .
$$

The above results are trivially generalized to the case of rotating charged $\mathrm{BH}$, see [3], for more detail.

Hence, due to the above equations we have verified that

$$
I_{b p s}=2 \pi f .
$$

\subsection{Relation between chemical potentials in the two formalisms}

At this point only reminds to understand the relation between the conjugated potentials in both pictures. In Sen's approach, the information about them is contained in the electric fields of the near horizon geometry, while in the Euclidean zero temperature formalism this information is encoded in the next to leading order term in an off-BPS expansion of the full geometry. Although these definitions seem to be rather different at first sight, notice that in Sen's approach the field strength is just the radial derivative of the potential evaluated at the horizon. In the Euclidean zero temperature case, the off-BPS expansion can be rewritten as an expansion in the radial position of the horizon $\rho_{+}$. Therefore, the next to leading order term in the off-BPS expansion of the gauge potential at $\rho_{+}$is proportional to its derivative with respect to the radial position of the horizon. Hence it is proportional to the field strength at the horizon. It is not dificult to check that the above reasoning produces exactly the result

$$
\phi_{i}=2 \pi e_{i} .
$$

therefore we have been able to relate all the different terms in the entropy functional approach with the emergent chemical potentials and thermodynamic functions of the zero temperature Euclidean approach.

\section{Conclusions}

As stated above, the main goal of this article is to provide a bridge between Sen's entropy functional formalism and standard Euclidean analysis of the thermodynamics of a black hole system. While doing so, we also find that the supergravity conjugated potentials defined in Sen's formalism map into chemical potentials of the dual CFT.

We obtain a unifying picture where:

1) We are able to recover the entropy function of Sen from the zero temperature limit of the usual $\mathrm{BH}$ thermodynamics and the statistical mechanics definitions of the dual CFT theory. The 
supergravity and their dual CFT chemical potentials are identified with the surviving Sen's near horizon electric and angular fields. The Euclidean action is identified with Sen's function $2 \pi f$.

2) As a byproduct of the above analysis we have understood how to calculate the BPS chemical potentials that control the statistical properties of the $\mathrm{BH}$ using only the BPS regime, i.e., without needing the knowledge of the non-BPS geometry. The CFT chemical potentials are dual to the supergravity ones. Traditionally, to compute the latter we have to start with the non-BPS solution and send the temperature to zero to find the next to leading order terms in the horizon angular velocities and electric potentials expansions that give the chemical potentials. This requires the knowledge of the non-BPS geometry. Unfortunately, sometimes this is not available and we only know the BPS solution. But, from item 1) we know that the near horizon fields, that Sen computes with the single knowledge of the BPS near horizon solution, give us the supergravity chemical potentials. So now we can compute the supergravity chemical potentials of any BPS BH solution, regardless of its embedding into a family of non-BPS solutions, while still keeping the relation with the dual CFT.

\section{Acknowledgments}

The author would like to thanks the organizers of the 3rd RTN Workshop for hospitality. This work was partially funded by the Ministerio de Educacion y Ciencia under grant FPA2005-02211 and CSIC via the I3P programme

\section{References}

[1] P. J. Silva, "Thermodynamics at the BPS bound for black holes in AdS," JHEP 0610, 022 (2006) arXiv:hep-th/0607056.

[2] P. J. Silva, "Phase transitions and statistical mechanics for BPS black holes in AdS/CFT," JHEP 0703, 015 (2007) arXiv:hep-th/0610163.

[3] O. J. C. Dias and P. J. Silva, "Euclidean analysis of the entropy functional formalism," arXiv:0704.1405 [hep-th].

[4] A. Sen, "Black hole entropy function and the attractor mechanism in higher derivative gravity," JHEP 0509 (2005) 038 arXiv:hep-th/0506177.

[5] A. Sen, "Entropy function for heterotic black holes," JHEP 0603 (2006) 008 arXiv:hep-th/0508042.

[6] D. Astefanesei, K. Goldstein, R. P. Jena, A. Sen and S. P. Trivedi, "Rotating attractors," JHEP 0610 (2006) 058 arXiv:hep-th/0606244.

[7] R. M. Wald, "Black hole entropy in the Noether charge," Phys. Rev. D 48 (1993) 3427 arXiv:gr-qc/9307038.

T. Jacobson, G. Kang and R. C. Myers, "On Black Hole Entropy," Phys. Rev. D 49 (1994) 6587 arXiv:gr-qc/9312023.

V. Iyer and R. M. Wald, "Some properties of Noether charge and a proposal for dynamical black hole entropy," Phys. Rev. D 50 (1994) 846 arXiv:gr-qc/9403028. 